\title{
Editorial
}

Dermatology

\section{Hidradenitis Suppurativa: The Disease Which Stimulates Researchers and Clinicians}

\author{
Jacek C. Szepietowski Łukasz Matusiak \\ Department of Dermatology, Venereology, and Allergology, Wroclaw Medical University, \\ Wroclaw, Poland
}

\section{Keywords}

Hidradenitis suppurativa · Acne inversa

Hidradenitis suppurativa (HS) is a chronic inflammatory skin disease of relapsing nature presenting with abscesses, nodules, and suppurating lesions mainly located in the intertriginous areas of the skin. Although there are divergent data coming from epidemiological studies, it is suggested that the disease affects around $1 \%$ of the general population [1]. HS is frequently misdiagnosed, especially in its early stages. A multicenter study clearly pointed out a 7.2-year delay in the correct diagnosis of HS. On average, the patients visit four physicians to have the final diagnosis established [2]. Patients suffer from pain and itch [3]. Moreover, HS is associated with comorbid diseases, named immune-mediated inflammatory disorders, such as colitis ulcerosa, Crohn's disease, seronegative arthritis, or pyoderma gangrenosum. Clinical manifestation, subjective symptoms, and malodor all contribute together to the profound burden of the disease. The quality of life in HS patients is heavily decreased; patients are stigmatized and develop secondary psychiatric disorders such as depression and anxiety, resulting in an increased risk of suicide [4]. The financial burden affects not only the sick individuum, but also the whole family. The etiopathogenesis of HS is not completely clear; it is multifactorial with several factors (i.e., genetic predisposition, hormonal and environmental ones) contributing to disease development and exacerbation. The complex nature of HS is a challenge for clinicians in the diagnostic process, assessing the disease severity and therapeutic approach. During recent years there has been huge progress in the treatment of HS, but effective treatment options still remain very limited [5]. We do think that HS represents one of the most important disease with unmet needs in the whole field of dermatology.

Almost three decades ago, when one of us (J.C.S.) began his career in dermatology, there was almost no interest in HS. The recurrent, suppurative, debilitating nature of HS with only few therapeutic options, quite commonly not satisfactory both for physicians and patients, created the atmosphere of undesirable disease in our daily clinical practice. That time only a few research groups concentrated on studies on the pathogenesis, clinical aspects, and therapeutic modalities in HS. Less than ten papers per year dealing with HS topics were published in the medical international literature. Nowadays, the situation is completely different. Due to new techniques in basic science allowing deeply studying the pathogenesis of HS,

\section{KARGER}

(c) 2020 S. Karger AG, Basel

E-Mail karger@karger.com

www.karger.com/drm
Jacek C. Szepietowski

Department of Dermatology, Venereology, and Allergology

Wroclaw Medical University

Chalubinskiego 1, PL-50-368 Wroclaw (Poland)

E-Mail jacek.szepietowski@umed.wroc.pl 
Fig. 1. The annual total number of publications on hidradenitis suppurativa/acne inversa in PubMed increased rapidly from 2013 to 2019.

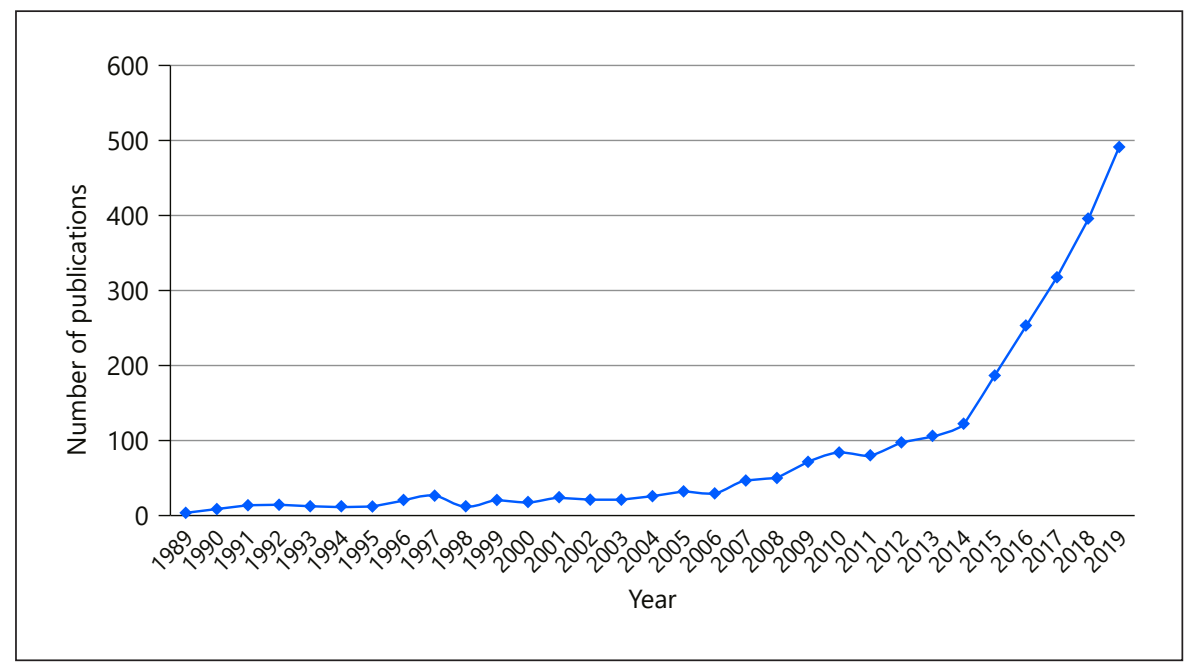

introducing new, more effective therapeutic options, we have recently observed an enormous increase in HS interest within the medical community. This became clear when we looked at the number of published papers (searched in PubMed with the key words: "hidradenitis suppurativa" and "acne inversa") during the last years. In 2013, this number for the first time reached 100 publications, in 2018 there were almost 400 papers published, and until December 2019 almost 500 scientific contributions were recorded in the PubMed database (Fig. 1).

It is important to underline that in 2005 the Hidradenitis Suppurativa Foundation, Inc. was created and in 2012 the European experts on HS decided to begin the European Hidradenitis Suppurativa Foundation (EHSF). Both those organizations grouped researchers and clinicians devoted to the field of HS and definitely stimulated basic and clinical studies in HS. In February 2018 we had the pleasure to welcome in Wroclaw, Poland HS experts from not only European countries, but also from the USA, Canada, Chile, China, and India for the 8th EHSF Conference. For the first time in the history of EHSF Conferences more than 400 participants created the atmosphere of an HS European celebration. During the three days of the meeting the plenary lectures were grouped into 14 scientific sessions. Moreover, 57 poster communications were presented. The 8th EHSF Conference was preceded by the European Academy of Dermatology and Venereology HS/Acne Inversa Course for specialists and residents in dermatology. Looking at the success of the above-mentioned conference and course, we are pleased that now we, a so numerous European group, work together to understand the interdisciplinary character of HS, and we are sure that all those efforts will result in increasingly better care for HS patients in the near future.
It is our honor to invite you to this special issue of Dermatology, which is composed of papers prepared based on communications presented during the 8th EHSF Conference in Wroclaw, Poland. We acknowledge Editor-inChief Prof. Dr. Gregor Jemec, world-class expert in HS, for creating this unique platform to present and discuss the hot topics in HS research. In this special issue you will find papers dealing with different aspects of HS. We would like to draw your attention to two papers focusing on the psychosocial burden of HS [6, 7]. Only a few years ago itch was reported and characterized in detail in HS [3, 8]. Here, we have the direct confirmation that both itch and malodor are important factors worsening the quality of life of HS patients. Interestingly, the authors documented that itch and malodor were linked with slightly different aspects of HS [6]. HS with its signs and symptoms, located frequently in anogenital areas, has a significant influence on patients' sexuality. Cuenca-Barrales and Molina-Leyva [7] showed that more than $50 \%$ of female HS patients presented with sexual dysfunction and that $60 \%$ of males with HS had erectile problems. Several scoring systems to assess the clinical severity of HS exist. Our group studied the interrater and intrarater reliability of the main HS scoring systems to answer the question whether residents in dermatology could adequately evaluate HS severity [9]. It seems crucial to educate our young colleagues on the most useful assessment tools for this disease. The treatment of HS is difficult, requires a holistic approach, and depends on clinical manifestation and staging of the disease. More than two decades ago biological agents were registered in selected immune-mediated inflammatory disorders. However, the first biological drug - adalimumab - was approved for HS patients by 
regulatory authorities only a few years ago. This year we reviewed the literature on biologics in HS, showing that many biological agents may be promising for HS patients and several new drugs are under development for this indication [10]. Moreover, it seems that individualized therapy for special groups of patients and/or special types of HS will be required. This is discussed by Zouboulis et al. [11], who propose dose intensification of adalimumab for recalcitrant HS. We also read with great interest a report on a series of HS patients controlled with ustekinumab [12]. Despite the newest therapeutics, we should also remember older modalities, such as intralesional injections of triamcinolone, which appeared beneficial for fistulous tracts in HS [13]. It is well known that disturbance in iron metabolism is associated with proinflammatory activation in many chronic diseases. To the best of our knowledge, the deranged iron status in HS patients was reported for the first time by Ponikowska et al. [14]. This could play a role in the inflammatory pathogenesis of HS. Katoulis et al. [15] discussed the role of increased colonization of HS patients with Staphylococcus aureus. They demonstrated that one-fourth of HS patients were colonized with $S$. aureus and that more than one-third of the strains were methicillin-resistant ones. The authors underlined the need for further microbiological studies in HS to clarify the clinical significance of their findings.
In summary, we hope that you, as we did, will enjoy reading papers in this special issue of Dermatology. We are sure that we will all meet again soon in Athens in 2020 for the next EHSF Conference to discuss new research results in $\mathrm{HS}$ and exchange our experience.

\section{Key Message}

HS is still a disease of unclear nature, integrating the research community for further extensive scientific investigations.

\section{Disclosure Statement}

This paper was not supported by any financial means. Ł. Matusiak has received honoraria from AbbVie, Pierre Fabre, and Polpharma for participation as a consultant and advisor as well as speaker honoraria from Leo Pharma, Medac, Novartis, and Valeant, and served as an investigator for AbbVie, Amgen, Behringer Ingelheim, Galapagos, InfraRX, Janssen, Menlo Therapeutics, Novartis, Pfizer, Regeneron, Trevi, and UCB. J.C. Szepietowski served as a consultant and advisor for AbbVie, Dignity Sciences, Leo Pharma, Novartis, Menlo Therapeutics, Pierre-Fabre, Sienna Biopharmaceuticals, and Trevi, as an investigator for AbbVie, Amgen, Behringer Ingelheim, Galapagos, InfraRX, Janssen, Leo Pharma, Menlo Therapeutics, Merck, Novartis, Regeneron, Trevi, and $\mathrm{UCB}$, and as a speaker for AbbVie, Janssen, Leo Pharma, Novartis, Sandoz, Sanofi-Genzyme, and Eli Lilly.

\section{References}

1 Zouboulis CC, Desai N, Emtestam L, Hunger RE, Ioannides D, Juhász I, et al. European S1 guideline for the treatment of hidradenitis suppurativa/acne inversa. J Eur Acad Dermatol Venereol. 2015 Apr;29(4):619-44.

2 Saunte DM, Boer J, Stratigos A, Szepietowski JC, Hamzavi I, Kim KH, et al. Diagnostic delay in hidradenitis suppurativa is a global problem. Br J Dermatol. 2015 Dec;173(6): $1546-9$.

3 Matusiak Ł, Szczęch J, Kaaz K, Lelonek E, Szepietowski JC. Clinical Characteristics of Pruritus and Pain in Patients with Hidradenitis Suppurativa. Acta Derm Venereol. 2018 Feb;98(2):191-4.

4 Matusiak $€$. Profound consequences of hidradenitis suppurativa: a review. Br J Dermatol. doi: 10.1111/bjd.16603 [Epub ahead of print].

5 Matusiak $€$, Jemec GB, Szepietowski JC. Pharmacological development in hidradenitis suppurativa. Curr Opin Pharmacol. 2019 Jun; 46:65-72.

6 Molina-Leyva A, Cuenca-Barrales C. Pruritus and malodour in patients with hidradenitis suppurativa: impact on quality of life and clinical features associated with symptom severity. Dermatology. doi: 10.1159/000502139 [Epub ahead of print].

7 Cuenca-Barrales C, Molina-Leyva A. Risk factors of sexual dysfunction in patients with hidradenitis suppurativa: a cross-sectional study. Dermatology. doi: 10.1159/000501905 [Epub ahead of print].

8 Vossen AR, Schoenmakers A, van Straalen KR, Prens EP, van der Zee HH. Assessing Pruritus in Hidradenitis Suppurativa: A CrossSectional Study. Am J Clin Dermatol. 2017 Oct;18(5):687-95.

9 Włodarek K, Stefaniak A, Matusiak Ł, Szepietowski JC. Could residents adequately assess the severity of hidradenitis suppurativa? Interrater and intrarater reliability assessment of major scoring systems. Dermatology. doi: 10.1159/000501771 [Epub ahead of print].

10 Włodarek K, Ponikowska M, Matusiak $€$, Szepietowski JC. Biologics for hidradenitis suppurativa: an update. Immunotherapy. 2019 Jan;11(1):45-59.

11 Zouboulis CC, Hansen H, Caposiena Caro RD, Damiani G, Delorme I, Pascual JC, et al. Adalimumab dose intensification in recalci- trant hidradenitis suppurativa/acne inversa. Dermatology. doi: 10.1159/000503606 [Epub ahead of print].

12 Romaní J, Vilarrasa E, Martorell A, Fuertes I, Ciudad C, Molina-Leyva A. Ustekinumab with intravenous infusion: results in hidradenitis suppurativa. Dermatology. doi: 10.1159/000501075 [Epub ahead of print].

13 Álvarez P, García-Martínez FJ, Poveda I, Pascual JC. Intralesional triamcinolone for fistulous tracts in hidradenitis suppurativa: an uncontrolled prospective trial with clinical and ultrasonographic follow-up. Dermatology. doi: 10.1159/000499934 [Epub ahead of print].

14 Ponikowska M, Matusiak L, Kasztura M Jankowska EA, Szepietowski JC. Deranged iron status evidenced by iron deficiency characterizes patients with hidradenitis suppurativa. Dermatology. 2020 Jan;236(1):51-7.

15 Katoulis A, Koumaki V, Efthymiou O, Koumaki D, Dimitroulia E, Voudouri M, et al. Staphylococcus aureus carriage status in patients with hidradenitis suppurativa: an observational cohort study in a tertiary referral hospital in Athens, Greece. Dermatology. doi: 10.1159/000504537 [Epub ahead of print].
Hidradenitis Suppurativa Stimulates

Researchers and Clinicians
Dermatology 2020;236:5-7

DOI: $10.1159 / 000505542$ 